\title{
FUNCTIONS OF $\Phi$-BOUNDED VARIATION AND RIEMANN-STIELTJES INTEGRATION ${ }^{1}$
}

\author{
BY
}

MICHAEL SCHRAMM

\begin{abstract}
A notion of generalized bounded variation is introduced which simultaneously generalizes many of those previously examined. It is shown that the class of functions arising from this definition is a Banach space with a suitable norm. Appropriate variation functions are defined and examined, and an analogue of Helly's theorem is estabished. The significance of this class to convergence of Fourier series is briefly discussed. A result concerning Riemann-Stieltjes integrals of functions of this class is proved.
\end{abstract}

\section{CHAPTER I}

We consider real valued functions whose domains are a closed, bounded interval, say $[a, b]$, to be made precise only if necessary. In the first section of this chapter we define a new notion of generalized bounded variation and the appropriate interval and point variation functions. We note some properties of functions which fall in this class, and observe how this new class is related to those already known. In $\S 2$ we examine the variation functions further, and investigate aspects of the linear structure of this class, showing that it is a Banach space and obtaining an analogue of Helly's theorem. In $\S 3$ we extend a theorem of Waterman concerning the convergence of Fourier series.

1. $\Phi$-bounded variation. Given a function $f$ and an interval $I=[x, y]$, we write $f(I)$ for $f(y)-f(x)$. Any collection $\left\{I_{n}\right\}$ of intervals mentioned shall be nonoverlapping subintervals of the domain of the function.

Let $\Phi=\left\{\phi_{n}\right\}$ be a sequence of increasing convex functions, defined on the nonnegative real numbers and such that $\phi_{n}(0)=0$ and $\phi_{n}(x)>0$ for $x>0$ and $n=1,2, \ldots$. We shall say that $\Phi$ is a $\Phi^{*}$-sequence if $\phi_{n+1}(x) \leq \phi_{n}(x)$ for all $n$ and $x$, and a $\Phi$-sequence if in addition $\sum_{n} \phi_{n}(x)$ diverges for $x>0$. If $\Phi$ is either a $\Phi^{*}$-sequence or a $\Phi$-sequence, we say that a function $f$ is of $\Phi$-bounded variation if the $\Phi$-sums $\sum_{n} \phi_{n}\left(\left|f\left(I_{n}\right)\right|\right)<\infty$ for any appropriate collection $\left\{I_{n}\right\}$. We denote by $\Phi B V$ the collection of all functions $f$ such that $c f$ is of $\Phi$-bounded variation for some $c>0$. In the present chapter all sequences used in this context will be assumed to be $\Phi$-sequences.

We begin with a lemma:

LEMMA 1.1. If the supremum of the $\Phi$-sums for a function $f$ is infinite, there is a point $x \in[a, b]$ such that the supremum of these sums remains infinite when

Received by the editors March 4, 1983; presented to the Society, April 16, 1982.

1980 Mathematics Subject Classification. Primary 26A45, 26A42; Secondary 42A20.

1 This paper is based on a doctoral dissertation written at Syracuse University under the direction of Professor Daniel Waterman. 
the subintervals considered are constrained to be any interval open relative to $[a, b]$ and containing $x$.

PROOF. Let $\left\{I_{n}\right\}$ be any collection of subintervals. Let $\left\{n_{k}\right\}$ be the set of integers with $n_{k}<n_{k+1}$ and $I_{n_{k}} \subset[a,(a+b) / 2]$ for all $k$, and let $\left\{m_{k}\right\}$ be the set of integers with $m_{k}<m_{k+1}$ and $I_{m_{k}} \subset[(a+b) / 2, b]$ for all $k$. Let $n_{*}$ be that integer, if any, such that $(a+b) / 2$ is an interior point of $I_{n_{*}}$. Note that $m_{k}, n_{k} \geq k$ for all $k$. Let $B=\sup f-\inf f$. Then

$$
\begin{aligned}
\sum_{n} \phi_{n}\left(\left|f\left(I_{n}\right)\right|\right) & =\sum_{k} \phi_{n_{k}}\left(\left|f\left(I_{n_{k}}\right)\right|\right)+\sum_{k} \phi_{m_{k}}\left(\left|f\left(I_{m_{k}}\right)\right|\right)+\phi_{n_{*}}\left(\left|f\left(I_{n_{*}}\right)\right|\right) \\
& \leq \sum_{k} \phi_{k}\left(\left|f\left(I_{n_{k}}\right)\right|\right)+\sum_{k} \phi_{k}\left(\left|f\left(I_{m_{k}}\right)\right|\right)+\phi_{1}(B) .
\end{aligned}
$$

The above inequality implies that if the $\Phi$-sums of $f$ are unbounded when the subintervals are chosen from all of $[a, b]$, then one half of the interval has the property that the $\Phi$-sums relative to it are unbounded. The last statement is valid for any interval over which the $\Phi$-sums are unbounded. Thus we may repeat the argument, obtaining a nested sequence of intervals whose lengths approach zero, each of which has this property. The intersection of these intervals is then a single point having the desired property.

THEOREM 1.2. The following are equivalent:

(i) $f$ is of $\Phi$-bounded variation:

(ii) there is an $M<\infty$ such that, for every $\left\{I_{n}\right\}, \sum_{n} \phi_{n}\left(\left|f\left(I_{n}\right)\right|\right)<M$;

(iii) there is an $M<\infty$ such that, for every finite collection $\left\{I_{n}\right\}$,

$$
\sum_{n} \phi_{n}\left(\left|f\left(I_{n}\right)\right|\right)<M
$$

ProOF. Clearly (ii) and (iii) are equivalent, and (ii) implies (i). We shall show that (i) implies (ii). Suppose that (ii) does not hold, and let $x$ be the point obtained by Lemma 1.1. Set $B=\sup f-\inf f$ and $J_{1}=[a, b]$, and let $\left\{I_{n}^{1}\right\}_{n=1}^{N_{1}}$ be a collection of subintervals of $J_{1}$ such that $\sum_{n=1}^{N_{1}} \phi_{n}\left(\left|f\left(I_{n}^{1}\right)\right|\right)>1+2 \phi_{1}(B)$. Upon deleting from this collection the interval(s) which contain $x$ and renumbering the remaining intervals if necessary, we obtain a collection $\left\{I_{n}^{1}\right\}_{n=1}^{N_{1}^{*}}$ (where $N_{1}^{*}=N_{1}, N_{1}-1$, or $N_{1}-2$ ) such that $\sum_{n=1}^{N_{i}^{*}} \phi_{n}\left(\left|f\left(I_{n}^{1}\right)\right|\right)>1$. Let $J_{2}$ be an interval, open relative to $J_{1}$ and containing $x$. such that $J_{2} \cap \bigcup_{1}^{N_{1}^{*}} I_{n}^{1}=\emptyset$. Let $\left\{I_{n}^{2}\right\}_{n=1}^{N_{2}}$ be a collection of subintervals of $J_{2}$ such that $N_{2}>N_{1}^{*}+2$ and $\sum_{n=N_{1}^{*}+1}^{N_{2}} \phi_{n}\left(\left|f\left(I_{n}^{2}\right)\right|\right)>1+2 \phi_{1}(B)$. Proceed as before to obtain $\sum_{n=N_{1}^{*}+1}^{N_{2}^{*}} \phi_{n}\left(\left|f\left(I_{n}^{2}\right)\right|\right)>1$, and $\sum_{k=1}^{2} \sum_{n=N_{k-1}^{*}+1}^{N_{k}^{*}} \phi_{n}\left(\left|f\left(I_{n}^{k}\right)\right|\right)>2$, where $N_{0}^{*}=0$. Continuing in this manner we obtain, for $m=1,2, \ldots$ and $k=1 \ldots, m$, collections of intervals $\left\{I_{n}^{k}\right\}_{k=1, n=N_{k-1}^{*}+1}^{m, N_{k}^{*}}$, such that

$$
\sum_{k=1}^{m} \sum_{n=N_{k-1}^{*}+1}^{N_{k}^{*}} \phi_{n}\left(\left|f\left(I_{n}^{k}\right)\right|\right)>m .
$$

Taking $\left\{I_{n}\right\}$ to be a renumbering in the obvious order of the union of these collections. we have $\sum_{n=1}^{\infty} \phi_{n}\left(\left|f\left(I_{n}\right)\right|\right)=\infty$. 
In view of Theorem 1.2 we may define, for $f$ of $\Phi$-bounded variation, the total $\Phi$-variation of $f: V_{\Phi}(f)=V_{\Phi}(f ; a, b)=\sup \sum_{n} \phi_{n}\left(\left|f\left(I_{n}\right)\right|\right)$, the supremum being taken over all $\left\{I_{n}\right\}, I_{n} \subseteq[a, b]$. We also define the $\Phi$-variation function of $f$, for $x \in[a, b]: v_{\Phi}(f ; x)=v_{\Phi}(x)=V_{\Phi}(f: a, x)$.

If $\Phi$ is a $\Phi$-sequence, it is clear that a function of $\Phi$-bounded variation is bounded and can have only simple discontinuities (hence only countably many of them). It is also easy to see that $\Phi B V$ is a linear set.

Various spaces of functions of generalized bounded variation which have been considered can be obtained by making special choices of the functions $\phi_{n}, n=$ $1,2, \ldots$. If we take $\phi_{n}(x)=x$ for all $n$, then $\Phi \mathrm{BV}=\mathrm{BV}$. If $\Lambda=\left\{\lambda_{n}\right\}$ is a $\Lambda$-sequence in the sense of Waterman [5], and we take $\phi_{n}(x)=x / \lambda_{n}$, we have $\Phi \mathrm{BV}=\Lambda \mathrm{BV}$. If $\Phi$ is an $N$-function in the sense of Young [7] and we let $\phi_{n}(x)=\phi(x)$ for all $n$, then $\Phi \mathrm{BV}=\phi \mathrm{BV}$. If $\phi$ is an $N$-function and $\Lambda$ is a $\Lambda$-sequence and we let $\phi_{n}(x)=\phi(x) / \lambda_{n}$, we obtain the space $\phi \Lambda \mathrm{BV}$, examined in [2 and $\mathbf{3}]$.

2. The space $\Phi B V$. We have said that $f \in \Phi B V$ if $V_{\Phi}(c f)<\infty$ for some $c>0$. Let us consider the expression $V_{\Phi}(c f)$ as a function of the variable $c$. If $\phi$ is an increasing convex function, $\phi(0)=0, x \geq 0$, and $0 \leq \alpha \leq 1$, we have $\phi(\alpha x) \leq \alpha \phi(x)$. Let $c_{1}>0$ be such that $V_{\Phi}\left(c_{1} f\right)<\infty$ and let $0<c \leq c_{1}$. Then $V_{\Phi}(c f) \leq\left(c / c_{1}\right) V_{\Phi}\left(c_{1} f\right) \rightarrow 0$ as $c \rightarrow 0$, in view of the previous comment. With this in mind, we define a norm as follows: Let $\Phi B V_{0}=\{f \in \Phi B V: f(a)=0\}$. For $f \in \Phi B V_{0}$, let $\|f\|=\inf \left\{k>0: V_{\Phi}(f / k) \leq 1\right\}$.

LEMMA 2.1. (i) $V_{\Phi}(f /\|f\|) \leq 1$;

(ii) if $\|f\| \leq 1$, then $V_{\Phi}(f) \leq\|f\|$.

ProOF. (i) Take $k>\|f\|$; then for any finite collection $\left\{I_{n}\right\}$,

$$
\sum_{n} \phi_{n}\left(\left|f\left(I_{n}\right)\right| / k\right) \leq V_{\Phi}(f / k) \leq 1
$$

Thus

$$
\sum_{n} \phi_{n}\left(\left|f\left(I_{n}\right)\right| /\|f\|\right)=\lim _{k \rightarrow\|f\|+} \sum_{n} \phi_{n}\left(\left|f\left(I_{n}\right)\right| / k\right) \leq 1,
$$

which implies (i).

(ii) For any $\left\{I_{n}\right\}$, since $\|f\| \leq 1$,

$$
\sum_{n} \phi_{n}\left(\left|f\left(I_{n}\right)\right|\right) \leq\|f\| \sum_{n} \phi_{n}\left(\left|f\left(I_{n}\right)\right| /\|f\|\right) \leq\|f\|,
$$

by (i).

THEOREM 2.2. $\|\cdot\|$ is a norm on $\Phi \mathrm{BV}_{0}$.

ProOF. (i) Since $\phi_{n}$ is increasing for each $n, V_{\Phi}\left(f / k_{1}\right) \leq V_{\Phi}(f / k)$ if $k_{1}>k$. Thus, by part (i) of Lemma 2.1, $\left\{k>0: V_{\Phi}(f / k) \leq 1\right\}=[\|f\|, \infty)$.

Clearly $\|0\|=0$. If $f \not \equiv 0$, let $x \in[a, b]$ be such that $f(x) \neq 0$. Then $V_{\Phi}(f / k) \geq$ $\phi_{1}(|f(x)| / k) \rightarrow \infty$ as $k \rightarrow 0$. Thus there is a $k>0$ so that $V_{\Phi}(f / k)>1$, and so $\|f\| \neq 0$

(ii)

$$
\begin{aligned}
\|c f\| & =\inf \left\{k>0: V_{\Phi}(c f / k) \leq 1\right\} \\
& =\inf \left\{k>0: V_{\Phi}(|c| f / k) \leq 1\right\}=|c|\|f\|
\end{aligned}
$$


(iii)

$$
\begin{aligned}
\sum_{n} \phi_{n}\left(\left|(f+g)\left(I_{n}\right)\right| /(\|f\|+\|g\|)\right) \leq & \sum_{n}(\|f\| /\|f\|+\|g\|) \phi_{n}\left(\left|f\left(I_{n}\right)\right| /\|f\|\right) \\
& +(\|g\| /\|f\|+\|g\|) \phi_{n}\left(\left|g\left(I_{n}\right)\right| /\|g\|\right) \leq 1,
\end{aligned}
$$

thus $\|f+g\| \leq\|f\|+\|g\|$.

THEOREM 2.3. $\left(\Phi \mathrm{BV}_{0},\|\cdot\|\right)$ is a Banach space.

PROOF. Let $f$ and $g$ be functions in $\Phi \mathrm{BV}_{0}$ such that $\|f-g\|<\varepsilon$. Then $\|(f-g) / \varepsilon\|<1$, so, by Lemma 2.1, $V_{\Phi}((f-g) / \varepsilon) \leq 1$. Now for $x \in[a, b]$,

$$
\phi_{1}(|f(x)-g(x)| / \varepsilon) \leq V_{\Phi}((f-g) / \varepsilon) \leq 1 .
$$

This implies that if $\left\{f_{n}\right\}$ is a Cauchy sequence in this norm it is also a Cauchy sequence in the supremum norm. Thus there is a function $f$ such that $f_{n} \rightarrow f$ uniformly. Let $\varepsilon>0$ be given, $\left\{I_{k}\right\}$ be a finite collection, and suppose $\left\|f_{n}-f_{m}\right\|<\varepsilon$, then

$$
\sum_{k} \phi_{k}\left(\left|\left(f_{n}-f\right)\left(I_{k}\right)\right| / \varepsilon\right)=\lim _{m \rightarrow \infty} \sum_{k} \phi_{k}\left(\left|\left(f_{n}-f_{m}\right)\left(I_{k}\right)\right| / \varepsilon\right) \leq 1 .
$$

Thus $V_{\Phi}\left(\left(f_{n}-f\right) / \varepsilon\right) \leq 1, f \in \Phi \mathrm{BV}$, and $f_{n} \rightarrow f$ in norm.

By Lemma 2.1, if $\left\|f_{n}-f\right\|<\varepsilon<1$, then $V_{\Phi}\left(f_{n}-f\right)<\varepsilon$ so that $f_{n} \rightarrow f$ in variation as well as in norm. Also note that convergence in norm implies uniform convergence, so that $\Phi \mathrm{BV}_{0} \cap C$ is also a Banach space with this norm. Finally, $\Phi B V$ may be made a Banach space with norm $|f(a)|+\|f-f(a)\|$.

We now examine the relationships between the continuity properties of the function $v_{\Phi}(f ; x)$ and those of $f$. The surprising complexity of these arguments illustrates some of the difficulties of the subject.

THEOREM 2.4. Let $f$ be of $\Phi$-bounded variation on $[a, b]$. Then:

(i) if $f$ is right continuous at $a, V_{\Phi}(f ; a, x) \rightarrow 0$ as $x \downarrow a$;

(ii) if $f$ is left continuous at $b, V_{\Phi}(f ; x, b) \rightarrow 0$ as $x \uparrow b$;

(iii) if $[x, y] \subseteq(a, b), V_{\Phi}(f ; x, y) \rightarrow 0$ as $x$ and $y$ together approach $a$ or $b$.

PROOF. It is clear that (i) and (ii) imply (iii), for if we set $g(x)=f(x)$ for $x \in(a, b), g(a)=f(a+)$ and $g(b)=f(b-)$, then $g$ is right continuous at $a$ and left continuous at $b$, so that

$$
0 \leq V_{\Phi}(f ; x, y)=V_{\Phi}(g ; x, y) \leq V_{\Phi}(g ; a, y) \rightarrow 0
$$

as $y \downarrow a$, and similarly for $x \uparrow b$.

We show (i). Let $I_{n}, n=1, \ldots, N_{1}$, be a collection of intervals such that $\left|f\left(I_{n}\right)\right| \downarrow, I_{n} \subseteq[a, b], f\left(I_{n}\right) \neq 0$ for each $n$ and

$$
\sum_{1}^{N_{1}} \phi_{n}\left(\left|f\left(I_{n}\right)\right|\right)>\frac{1}{2} V_{\Phi}(f ; a, b) .
$$

Since $f$ is right continuous at $a$ and each $\phi_{n}$ is continuous, we may assume that $I_{n} \subseteq(a, b]$ for each $n$. Choose $y_{1} \in(a,(a+b) / 2)$ such that $\left[a, y_{1}\right] \cap \bigcup_{1}^{N_{1}} I_{n}=\emptyset$ 
and $I \subseteq\left(a, y_{1}\right]$ implies $|f(I)| \leq\left|f\left(I_{N_{1}}\right)\right|$. There is a collection of intervals $I_{n}, n=$ $N_{1}+1, \ldots, N_{2}$, with $\left|f\left(I_{n}\right)\right| \downarrow, I_{n} \subseteq\left(a, y_{1}\right], f\left(I_{n}\right) \neq 0$ for each $n$, and

$$
\sum_{N_{1}+1}^{N_{2}} \phi_{n-N_{1}}\left(\left|f\left(I_{n}\right)\right|\right)>\frac{1}{2} V_{\Phi}\left(f ; a, y_{1}\right) \text {. }
$$

Continuing in this manner we obtain, for $k=1,2, \ldots$, points $y_{k}$ and intervals $I_{N_{k}+1}, \ldots, I_{N_{k+1}}$ contained in $\left[y_{k+1}, y_{k}\right]$, such that $y_{k} \downarrow a,\left|f\left(I_{n}\right)\right| \downarrow$, and

$$
\sum_{N_{k}+1}^{N_{k+1}} \phi_{n-N_{k}}\left(\left|f\left(I_{n}\right)\right|\right)>\frac{1}{2} V_{\Phi}\left(f ; a, y_{k}\right) .
$$

Since $f$ is of $\Phi$-bounded variation, $\sum_{1}^{\infty} \phi_{n}\left(\left|f\left(I_{n}\right)\right|\right)<\infty$. Thus, given $\varepsilon>0$, there is an $N$ so that

$$
\sum_{N+1}^{\infty} \phi_{n}\left(\left|f\left(I_{n}\right)\right|\right)<\varepsilon / 2 .
$$

Since $\left|f\left(I_{n}\right)\right| \downarrow 0, \sum_{N+1}^{\infty} \phi_{n}\left(\left|f\left(I_{n+j}\right)\right|\right)<\varepsilon / 2$ for any $j>0$. Further, there is a $J_{\varepsilon}$ such that $\sum_{1}^{N} \phi_{n}\left(\left|f\left(I_{n+j}\right)\right|\right)<\varepsilon / 2$ for $j>J_{\varepsilon}$. Taking $j=N_{k}$, we have

$$
\frac{1}{2} V_{\Phi}\left(f ; a, y_{k}\right)<\sum_{1}^{\infty} \phi_{n}\left(\left|f\left(I_{n+N_{k}}\right)\right|\right)<\varepsilon
$$

for $k$ sufficiently large. Hence $V_{\Phi}\left(f ; a, y_{k}\right) \rightarrow 0$ as $k \rightarrow \infty$, and so $V_{\Phi}(f ; a, y) \rightarrow 0$ as $y \downarrow a$, since $V_{\Phi}(f ; a, y)$ is a monotone function of $y$.

In the next lemma we use the following notation: For a given $\Phi$-sequence $\Phi$, and $\delta>0$, let

$$
\begin{aligned}
& k(M, \delta)=\min \left\{n: \sum_{1}^{n} \phi_{j}(\delta / 2)>M\right\} ; \\
& \beta(k, \delta)=\min \left\{1-\left(\phi_{j}(\delta / 2) / \phi_{j}(\delta)\right): 1 \leq j \leq k\right\} .
\end{aligned}
$$

Note that $M_{1}>M$ implies $k\left(M_{1}, \delta\right) \geq k(M, \delta), k_{1}>k$ implies $\beta\left(k_{1}, \delta\right) \leq \beta(k, \delta)$, and $\beta(k, \delta)>0$ for any $k$ and $\delta$.

LEMMA 2.5. If $f$ is of $\Phi$-bounded variation and $[x, y] \subseteq[a, b]$ is such that $|f([x, y])| \geq \delta>0$, then

$$
v_{\Phi}(y)-v_{\Phi}(x) \geq \beta_{0} \phi_{k_{0}}(\delta),
$$

where $k_{0}=k\left(v_{\Phi}(x), \delta\right)$ and $\beta_{0}=\beta\left(k_{0}, \delta\right)$.

Proof. Given $\eta>0$, there exist $I_{n}, n=1, \ldots, N, I_{n} \subseteq[a, x]$, such that $\left|f\left(I_{n}\right)\right| \downarrow$ and $v_{\Phi}(x) \leq \sum_{1}^{N} \phi_{n}\left(\left|f\left(I_{n}\right)\right|\right)+\eta$. Let

$$
m=\min \left(\left\{n:\left|f\left(I_{n}\right)\right|<\delta / 2\right\} \cup\{N+1\}\right) .
$$

We show

$$
v_{\Phi}(y)-v_{\Phi}(x) \geq \phi_{m}(\delta)-\phi_{m}(\delta / 2)-\eta
$$

Let $a_{n}=\left|f\left(I_{n}\right)\right|$ and $T=\sum_{1}^{N} \phi_{n}\left(a_{n}\right)$. We construct a second sum, $S$, and compute $S-T$ as follows. 
(1) If $a_{n} \geq \delta / 2$ for $n=1, \ldots, k$ and $a_{k+1}<\delta / 2$, set $S=\phi_{1}\left(a_{1}\right)+\cdots+\phi_{k}\left(a_{k}\right)+$ $\phi_{k+1}(\delta)+\phi_{k+2}\left(a_{k+1}\right)+\cdots+\phi_{N+1}\left(a_{N}\right)$. Then $S-T \geq \phi_{k+1}(\delta)-\phi_{k+1}(\delta / 2)$;

(2) if $a_{n} \geq \delta / 2$ for $n=1, \ldots, N$, set $S=T+\phi_{N+1}(\delta / 2)$;

(3) if $a_{n}<\delta / 2$ for $n=1, \ldots, N$, set $S=\phi_{1}(\delta)+\phi_{2}\left(a_{1}\right)+\cdots+\phi_{N+1}\left(a_{N}\right)$. Then $S-T \geq \phi_{1}(\delta)-\phi_{1}(\delta / 2)$.

The sum $S$ serves as a lower approximation to $v_{\Phi}(y)$, this construction assuring that the quantity $f([x, y])$ enters the approximation in an effective manner. In any case, $v_{\Phi}(y)-v_{\Phi}(x) \geq S-T-\eta$. Thus,

$$
v_{\Phi}(y)-v_{\Phi}(x) \geq \begin{cases}\phi_{k+1}(\delta)-\phi_{k+1}(\delta / 2) & \text { if } a_{k+1}<\delta / 2 \leq a_{k} \\ \phi_{N+1}(\delta)-\phi_{N+1}(\delta / 2) & \text { if } a_{n} \geq \delta / 2 \text { for all } n \\ \phi_{1}(\delta)-\phi_{1}(\delta / 2) & \text { if } a_{n}<\delta / 2 \text { for all } n\end{cases}
$$

which is $(*)$, implying

$$
v_{\Phi}(y)-v_{\Phi}(x) \geq\left(1-\left(\phi_{m}(\delta / 2) / \phi_{m}(\delta)\right)\right) \phi_{m}(\delta)-\eta .
$$

Now if $m=1$, obviously $k_{0} \geq m$. If $m=N+1$,

$$
v_{\Phi}(x) \geq \sum_{1}^{N} \phi_{n}\left(a_{n}\right) \geq \sum_{1}^{N} \phi_{n}(\delta / 2)
$$

so that $k_{0} \geq N+1$. If $1<m<N+1$,

$$
v_{\Phi}(x) \geq \sum_{1}^{m-1} \phi_{n}\left(a_{n}\right) \geq \sum_{1}^{m-1} \phi_{n}(\delta / 2)
$$

so that $k_{0}>m+1$. In any case, $k_{0} \geq m$, so that

$$
\left(1-\left(\phi_{m}(\delta / 2) / \phi_{m}(\delta)\right)\right) \phi_{m}(\delta)-\eta \geq \beta_{0} \phi_{k_{0}}(\delta)-\eta
$$

and since $\eta$ was arbitrary and $\beta_{0}$ and $k_{0}$ do not depend on $\eta$, we have $v_{\Phi}(y)-$ $v_{\Phi}(x) \geq \beta_{0} \phi_{k_{0}}(\delta)$.

LEMMA 2.6. If $\Phi=\left\{\phi_{n}\right\}$ is a $\Phi^{*}$-sequence, then $\Phi$ is equicontinuous over any interval $[0, B], B<\infty$.

PROOF. By an elementary property of convex functions we have, for $y>x$ and each $n$,

$$
\begin{aligned}
\left(\phi_{n}(y)-\phi_{n}(x)\right) /(y-x) & \leq \phi_{n}(y+1)-\phi_{n}(y) \\
& <\phi_{n}(y+1) \leq \phi_{1}(y+1),
\end{aligned}
$$

which implies the desired result.

THEOREM 2.7. Let $f$ be of $\Phi$-bounded variation. Then $v_{\Phi}$ is right (left) continuous at a point $x \in[a, b]$ if and only if $f$ is right (left) continuous at $x$.

ProOF. We consider only the case of right continuity. Since $\phi_{k_{0}}(x)>0$ for $x>0\left(k_{0}\right.$ as in Lemma 2.5), that the right continuity of $v_{\Phi}$ implies right continuity of $f$ is a direct consequence of Lemma 2.5. To establish the opposite implication, suppose that $v_{\Phi}$ is not right continuous at a point $x \in[a, b)$ but what $f$ is right continuous at $x$. Then there is a $\delta>0$ so that $v_{\Phi}(y)-v_{\Phi}(x)>\delta$ fo: all $y>x$. Let $\omega$ be the modulus of equicontinuity of $\Phi$ on $[0, \sup f-\inf f]$, and choose $y>x$ so that $\omega(\operatorname{osc}(f,[x, y]))<\delta / 3$. Let $I_{n} \subseteq[a, y], n=1,2, \ldots$ Let $\left\{n_{k}\right\}$ be the increasing set 
of indices such that $I_{n_{k}} \subseteq[a, x],\left\{m_{k}\right\}$ be the increasing set of indices such that $I_{m_{k}} \subseteq[x, y]$, and $n_{*}$ that index, if any, such that $x$ is an interior point of $I_{n_{*}}$. Then we have

$$
\begin{aligned}
\sum_{n} \phi_{n}\left(\left|f\left(I_{n}\right)\right|\right) & =\sum_{k} \phi_{n_{k}}\left(\left|f\left(I_{n_{k}}\right)\right|\right)+\sum_{k} \phi_{m_{k}}\left(\left|f\left(I_{m_{k}}\right)\right|\right)+\phi_{n_{*}}\left(\left|f\left(I_{n_{*}}\right)\right|\right) \\
& =S_{1}+S_{2}+\phi_{n_{*}}\left(\left|f\left(I_{n_{*}}\right)\right|\right) .
\end{aligned}
$$

We may choose $\left\{I_{n}\right\}$ so that $v_{\Phi}(y)-\sum_{n} \phi_{n}\left(\left|f\left(I_{n}\right)\right|\right)<\delta / 3$. Then $\sum_{n} \phi_{n}\left(\left|f\left(I_{n}\right)\right|\right)-$ $v_{\Phi}(x)>2 \delta / 3$. Setting $I^{\prime}=I_{n_{*}} \cap[a, x]$, we have $\left|\phi_{n_{*}}\left(\left|f\left(I_{n_{*}}\right)\right|\right)-\phi_{n_{*}}\left(\left|f\left(I^{\prime}\right)\right|\right)\right|<\delta / 3$, so that

$$
\left(S_{1}+\phi_{n *}\left(f\left(I^{\prime}\right) \mid\right)-v_{\Phi}(x)\right)+S_{2}>\delta / 3 .
$$

Now the expression in parentheses is not positive, which implies that $S_{2}>\delta / 3$. But $V_{\Phi}(f ; x, y) \geq S_{2}$, and so $V_{\Phi}(f ; x, y)>\delta / 3$, which contradicts Theorem 2.4.

We will use the preceding results to establish the following analogue of the Helly Selection Theorem:

THEOREM 2.8. If for $\left\{f_{n}\right\}_{n=1}^{\infty} \subseteq \Phi \mathrm{BV}$ there is a $c>0$ and an $M<\infty$ such that $\left|c f_{n}(x)\right|<M$ for all $n$ and $x \in[a, b]$, and $V_{\Phi}(c f)<M$ for all $n$, then there is a subsequence $\left\{f_{n_{k}}\right\} \subseteq\left\{f_{n}\right\}$ and a function $f \in \Phi \mathrm{BV}$ so that $f_{n_{k}}(x) \rightarrow f(x)$ for all $x \in[a, b]$ and $V_{\Phi}(c f) \leq M$.

PROOF. Set $v_{n}(x)=V_{\Phi}\left(c f_{n} ; a, x\right), n=1,2, \ldots$ Then $v_{n}$ is nondecreasing for all $n$ and $0 \leq v_{n}(x) \leq M$ for all $n$ and $x \in[a, b]$. Thus $v_{n} \in \mathrm{BV}$ for all $n$, and we may apply the Helly Selection Theorem to $\left\{v_{n}\right\}$ to obtain a subsequence $\left\{v_{n_{k}}\right\}$ and a function $v$ so that $v_{n_{k}}(x) \rightarrow v(x)$ for all $x \in[a, b], v$ is nondecreasing, and $0 \leq v(x) \leq M$ for all $x \in[a, b]$. By a diagonalization process, we may find $\left\{f^{j}\right\}$, a subsequence of $\left\{f_{n_{k}}\right\}$ such that $\left\{f^{j}(x)\right\}$ converges at $a, b$, and all rational points of $[a, b]$. Let $\left\{v^{j}\right\}$ be the corresponding subsequence of $\left\{v_{n_{k}}\right\}$.

Since $v$ is nondecreasing, it is continuous for almost all $x \in[a, b]$. Let $x_{0} \in(a, b)$ be an irrational point at which $v$ is continuous. There is a rational $y>x_{0}, y \in(a, b)$ such that

$$
0 \leq v(y)-v\left(x_{0}\right)<\eta=\beta_{1} \phi_{k_{1}}(\varepsilon) / 3,
$$

where $k_{1}=k(M, \varepsilon)$ and $\beta_{1}=\beta\left(k_{1}, \varepsilon\right)$. There is an integer $J$ such that, for $j<J,\left|v^{j}(y)-v(y)\right|<\eta$ and $\left|v^{j}\left(x_{0}\right)-v\left(x_{0}\right)\right|<\eta$. Then, for $j<J$,

$$
0 \leq v^{j}(y)-v^{j}\left(x_{0}\right)<3 \eta=\beta_{1} \phi_{k_{1}}(\varepsilon) \leq \beta_{0} \phi_{k_{0}}(\varepsilon),
$$

where $k_{0}=k\left(v^{j}\left(x_{0}\right), \varepsilon\right)$ and $\beta_{0}=\beta\left(k_{0}, \varepsilon\right)$. The last inequality is valid due to the comments following the definitions of $k(M, \varepsilon)$ and $\beta(k, \varepsilon)$, since $v^{j}\left(x_{0}\right) \leq M$. By Lemma 2.5, for $j>J,\left|f^{j}(y)-f^{j}\left(x_{0}\right)\right|<\varepsilon$. Since $\left\{f^{j}(y)\right\}$ converges, there is an integer $J^{\prime}$ such that, for $j, k>J^{\prime},\left|f^{j}(y)-f^{k}(y)\right|<\varepsilon$, and so, for $j, k>\max \left\{J, J^{\prime}\right\}$,

$$
\left|f^{j}\left(x_{0}\right)-f^{k}\left(x_{0}\right)\right|<3 \varepsilon
$$

and thus $\left\{f^{j}\left(x_{0}\right)\right\}$ converges. So $\left\{f^{j}(x)\right\}$ converges except possibly on a countable set (the set of discontinuities of $v$ ). By another diagonalization we may find a subsequence $\left\{f_{(i)}\right\} \subseteq\left\{f^{j}\right\}$ so that $\left\{f_{(i)}(x)\right\}$ converges for all $x \in[a, b]$. Let $\left\{v_{(i)}\right\}$ be the corresponding subsequence of $\left\{v^{j}\right\}$, and let $f(x)=\lim _{i \rightarrow \infty} f_{(i)}(x)$. 
Let $I_{n} \subseteq[a, b], n=1, \ldots, N$. For any $\varepsilon>0$, there is an integer $J$ such that, for $i>J, v(b)>v_{(i)}(b)-\varepsilon$ and

$$
\left|\sum_{n=1}^{N} \phi_{n}\left(c\left|f_{(i)}\left(I_{n}\right)\right|\right)-\sum_{n=1}^{N} \phi_{n}\left(c\left|f\left(I_{n}\right)\right|\right)\right|<\varepsilon .
$$

Then, for $i>J$,

$$
\begin{aligned}
M \geq v(b)>v_{(i)}(b)-\varepsilon & \geq \sum_{n=1}^{N} \phi_{n}\left(c\left|f_{(i)}\left(I_{n}\right)\right|\right)-\varepsilon \\
& >\sum_{n=1}^{N} \phi_{n}(c|f(I)|)-2 \varepsilon
\end{aligned}
$$

which implies that $V_{\Phi}(c f) \leq M$.

3. Fourier series. The Dirichlet-Jordan theorem states that if $f$ is a function of bounded variation on $[a, b]$ the Fourier series of $f$ converges for all $x \in[a, b]$ and that this convergence is uniform over any closed interval of points of continuity of $f$. The desire to extend this theorem to larger classes of functions has provided much of the impetus for the study of generalizations of bounded variation. By taking $\phi_{n}(x)=x / n$, we obtain the class of functions of harmonic bounded variation (HBV). Waterman $[\mathbf{4}, \mathbf{5}]$ showed that the conclusion of the Dirichlet-Jordan theorem holds for $f \in \mathrm{HBV}$, and that this is best possible in the sense that if $\Lambda \mathrm{BV}$ is strictly larger than $\mathrm{HBV}$ there is a continuous function in $\Lambda \mathrm{BV}$ whose Fourier series diverges at a point. We extend this result as follows:

THEOREM 3.1. Let $[a, b]=[0,2 \pi]$. If $\mathrm{HBV}$ is properly contained in $\Phi \mathrm{BV}$, there is a continuous function in $\mathrm{BBV}_{0}$ whose Fourier series diverges at $x=0$.

PROOF. If HBV is properly contained in $\Phi \mathrm{BV}$, there exists a sequence $\left\{\alpha_{n}\right\}$ with $\alpha_{n} \geq \alpha_{n+1} \rightarrow 0, \sum_{n} \phi_{n}\left(\alpha_{n}\right)<\infty$ and $\sum_{n} \alpha_{n} / n=\infty$. Let $\beta_{1}=\beta_{2}=\alpha_{2}$, $\beta_{3}=\beta_{4}=\alpha_{4}$, etc. Then, since $\beta_{i} \leq \alpha_{i}, i=1,2, \ldots$, we have $\sum_{n} \phi_{n}\left(\beta_{n}\right)<\infty$ while $\sum_{n} \beta_{n} / n \geq \sum_{n} \alpha_{2 n} / 2 n=\infty$. Choose $\varepsilon>0$ so that $\sum_{n} \phi_{n}\left(\varepsilon \beta_{n}\right)<1$, and let $a_{n}=\varepsilon \beta_{n}$. Then

(1) $a_{n} \geq a_{n+1} \rightarrow 0$

(2) $a_{2 n-1}=a_{2 n}, n=1,2, \ldots$, and

(3) $\sum_{n} \phi_{n}\left(a_{n}\right)<1$ and $\sum_{n} a_{n} / n=\infty$.

Let

$$
f_{n}(x)= \begin{cases}a_{i}, & (2 i-2) \pi<\left(n+\frac{1}{2}\right) x<(2 i-1) \pi, i=1, \ldots, n+1 \\ 0, & \text { otherwise }\end{cases}
$$

Then $\sup \sum_{k} \phi_{k}\left(\left|f_{n}\left(I_{k}\right)\right|\right)$ is achieved by choosing $\left\{I_{k}\right\}$ so that $\left|f_{n}\left(I_{k}\right)\right|=a_{k}, k=$ $1, \ldots, n+1$. When $\left\{I_{k}\right\}$ is chosen in this manner, $V_{\Phi}\left(f_{n}\right)=\sum_{k} \phi_{k}\left(\left|f_{n}\left(I_{k}\right)\right|\right)=$ $\sum_{k} \phi_{k}\left(a_{k}\right)<1$, so that $f_{n} \in \Phi \mathrm{BV}_{0}$ and $\left\|f_{n}\right\|=\inf \left\{k: V_{\Phi}\left(f_{n} / k\right) \leq 1\right\} \leq 1$. 
Let $S_{n}(f)$ be the $n$th partial sum of the Fourier series of $f$ at $x=0$, and let $D_{n}(t)=\left(\sin \left(n+\frac{1}{2}\right) t\right) / 2 \sin \frac{1}{2} t$ be the Dirichlet kernel. Then

$$
\begin{aligned}
\pi S_{n}\left(f_{n}\right) & =\int_{0}^{2 \pi} f(t) D_{n}(t) d t \\
& \geq \int_{0}^{2 \pi} f_{n}(t)\left(\sin \left(n+\frac{1}{2}\right) t / t\right) d t \\
& =\sum_{i=1}^{n+1} \int_{(2 i-2) \pi /(n+1 / 2)}^{(2 i-1) \pi /(n+1 / 2)}\left(f_{n}(t) \sin \left(n+\frac{1}{2}\right) t / t\right) d t \\
& \geq \sum_{i=1}^{n+1} a_{i}\left(\left(n+\frac{1}{2}\right) /(2 i-1) \pi\right)\left(2 /\left(n+\frac{1}{2}\right)\right) \\
& >\frac{1}{\pi} \sum_{i=1}^{n+1} a_{i} / i \rightarrow \infty \quad \text { as } n \rightarrow \infty
\end{aligned}
$$

Without changing their variations or norms, the functions $f_{n}, n=1,2, \ldots$, may be altered on a set of suitably small measure so that they are continuous and the partial sums of their Fourier series differ from those of the functions $f_{n}$ by a small amount. Recalling that $\Phi B V_{0} \cap C$ is a Banach space, the proof is complete by the uniform boundedness principle.

\section{CHAPTER II}

1. In this chapter we obtain analogues of theorems of Young [6] and Leśniewicz and Orlicz [1] concerning Riemann-Stieltjes integration. We follow the technique of Leśniewicz and Orlicz, and each of these results is an extension of their work, though the proofs of some of the lemmas have been shortened and clarified. In this chapter the nature of each sequence $\Phi$ will be specified.

LEMMA 1.1. If $\Phi=\left\{\phi_{n}\right\}$ and $\Psi=\left\{\psi_{n}\right\}$ are $\Phi^{*}$-sequences,

$$
\sum_{k} \phi_{k}^{-1}(1 / k) \psi_{k}^{-1}(1 / k)<\infty
$$

and $A, B \geq 0$, then $\sum_{k} \phi_{k}^{-1}(A / k) \psi_{k}^{-1}(B / k)<\infty$.

PROOF. Let $m$ be a positive integer such that $A \leq m$ and $B \leq m$. Then, for $i m \leq k<(i+1) m$, we have $A / k \leq 1 / i$ and $B / k \leq 1 / i$, so that

$$
\begin{aligned}
\sum_{k} \phi_{k}^{-1}(A / k) \psi_{k}^{-1}(B / k) \leq & \sum_{1}^{m-1} \phi_{k}^{-1}(A / k) \psi_{k}^{-1}(B / k) \\
& +m \sum_{i=1}^{\infty} \phi_{i}^{-1}(1 / i) \psi_{i}^{-1}(1 / i)<\infty .
\end{aligned}
$$

LEMMA 1.2. If $\sum_{k} \phi_{k}^{-1}(1 / k) \psi_{k}^{-1}(1 / k)<\infty$, there exists a convex function $F:[0, \infty) \rightarrow[0, \infty)$ with $F(0)=0, F(x)>0$ for $x>0$, and $F(x)=o(x)$ as $x \rightarrow 0$ such that $\sum_{k}\left(F \phi_{k}\right)^{-1}(1 / k)\left(F \psi_{k}\right)^{-1}(1 / k)<\infty$.

Proof. By Lemma 1.1, $\sum_{k} \phi_{k}^{-1}(3 n / k) \psi_{k}^{-1}(3 n / k)<\infty$ for $n=1,2, \ldots$. Thus we may choose $\left\{k_{n}\right\}$, a sequence of positive integers, so that $k_{n+1}>(1+1 / n) k_{n}$ 
and

Set

$$
\sum_{k=k_{n}}^{\infty} \phi_{k}^{-1}(3 n / k) \psi_{k}^{-1}(3 n / k)<1 / n^{2} \quad \text { for } n=1,2, \ldots
$$

$$
p(t)= \begin{cases}\frac{1}{n}, & \frac{1}{k_{n+1}}<t \leq \frac{1}{k_{n}} \\ 1+t, & \frac{1}{k_{1}}<t\end{cases}
$$

Then $p(t)$ is a positive, increasing function of $t$ and $p(t) \rightarrow 0$ as $t \rightarrow 0$. Let $F(x)=\int_{0}^{x} p(t) d t$ for $x \geq 0$. Then $F$ is convex, $F(0)=0$, and $F(x)=o(x)$ as $x \rightarrow 0$. We will see that $F$ has the desired property. If $n k_{n}<k<n k_{n+1}$, we have

$$
F(3 n / k) \geq \int_{n / k}^{2 n / k} p(t) d t \geq(n / k) p(n / k) \geq 1 / k .
$$

Also, if $n k_{n+1} \leq k<(n+1) k_{n+1}$, we have

$$
F(3 n / k) \geq \int_{(n+1) / k}^{(2 n+1) / k} p(t) d t \geq(n / k) p((n+1) / k)=1 / k,
$$

so that $F(3 n / k) \geq 1 / k$ for $n k_{n} \leq k<(n+1) k_{n+1}$, and so $F^{-1}(1 / k) \leq 3 n / k$ for $k$ in this interval. Thus

$$
\begin{aligned}
& \sum_{k}\left(F \phi_{k}\right)^{-1}(1 / k)\left(F \psi_{k}\right)^{-1}(1 / k) \\
&=\sum_{k=1}^{k_{1}-1} \phi_{k}^{-1} F^{-1}(1 / k) \psi_{k}^{-1} F^{-1}(1 / k) \\
&+\sum_{n=1}^{\infty} \sum_{k=n k_{n}}^{(n+1) k_{n+1}-1} \phi_{k}^{-1} F^{-1}(1 / k) \psi_{k}^{-1} F^{-1}(1 / k) \\
& \leq C+\sum_{n=1}^{\infty} \sum_{k=n k_{n}}^{(n+1) k_{n+1}-1} \phi_{k}^{-1}(3 n / k) \psi_{k}^{-1}(3 n / k) \\
& \leq C+\sum_{n=1}^{\infty} \sum_{k=k_{n}}^{\infty} \phi_{k}^{-1}(3 n / k) \psi_{k}^{-1}(3 n / k) \\
& \leq C+\sum_{n=1}^{\infty} 1 / n^{2}<\infty .
\end{aligned}
$$

Now suppose $F$ is a convex function with $F(x)=o(x)$ as $x \rightarrow 0$ (we will actually use only the particular $F$ constructed in Lemma 1.2). If $\Phi=\left\{\phi_{n}\right\}$, let $F \Phi=\left\{F \phi_{n}\right\}$. Note that $F \Phi$ may not be a $\Phi$-sequence, since we need not have $\sum_{n} F \phi_{n}(x)=\infty$ for all $x>0$. Nevertheless, we may define $V_{F \Phi}(f)$ and $F \Phi B V$ in the usual way, observing that some of our results may not be valid for this "variation" and this class. Since $F(x)=o(x)$, we have $F \phi_{n}(x)=o\left(\phi_{n}(x)\right)$. This implies that $\Phi \mathrm{BV} \subseteq$ $F \Phi \mathrm{BV}$, more precisely, $V_{F \Phi}(f)<\infty$ if $V_{\Phi}(f)<\infty$. For a $\Phi$-sequence, $\Phi$, let $\chi_{n}(\Phi, x)=F\left(\phi_{n}(x)\right) / \phi_{n}(x)$ for $x>0$, and $\chi_{n}(\Phi, 0)=0$. Taking into account the properties of $\Phi$-sequences and the function $F$, we observe that:

(i) $\chi_{n}(\Phi, x)$ is nondecreasing;

(ii) $\chi_{n+1}(\Phi, x) \geq \chi_{n}(\Phi, x)$ for all $n$; and

(iii) $\chi_{n}(\Phi, x) \rightarrow 0$ as $x \rightarrow 0$. 
LEMMA 1.3. Let $B(f)=\sup f-\inf f$. Then

(i) $\phi_{1}(B(f)) \leq V_{\Phi}(f)$,

(ii) $V_{F \Phi}(f) \leq \chi_{1}(\Phi, B(f)) V_{\Phi}(f)$.

Proof. Part (i) is clear.

(ii)

$$
\begin{aligned}
V_{F \Phi}(f) & =\sup \sum_{n} F \phi_{n}\left(\left|f\left(I_{n}\right)\right|\right) \\
& =\sup \sum_{n} \chi_{n}\left(\Phi,\left|f\left(I_{n}\right)\right|\right) \phi_{n}\left(\left|f\left(I_{n}\right)\right|\right) \\
& \leq \chi_{1}(\Phi, B(f)) V_{\Phi}(f) .
\end{aligned}
$$

(Part (ii) of this lemma quantifies the remark made above concerning $V_{F \Phi}(f)$ and $V_{\Phi}(f)$.)

We now introduce some notation which will simplify our computations. Let $\Phi=\left\{\phi_{k}\right\}$ be a $\Phi^{*}$-sequence, $a=\left\{a_{k}\right\}$ a sequence of real numbers, and $\delta=\left\{\delta_{k}\right\}$ a sequence of integers with $0 \leq \delta_{0}<\delta_{1}<\cdots$.

Define

(1) $\rho_{n}(\Phi, a)=\sum_{k=1}^{n} \phi_{k}\left(\left|a_{k}\right|\right)$;

(2) $\delta(a)=\left\{\delta(a)_{n}\right\}$ by $\delta(a)_{n}=\sum_{k=\delta_{n-1}+1}^{\delta_{n}} a_{k}$; and

(3) $\rho_{n}^{*}(\Phi, a)=\sup _{\delta} \rho_{n}(\Phi, \delta(a))$.

LEMMA 1.4. For any sequence $a=\left\{a_{k}\right\}$ and $\Phi^{*}$-sequence $\Phi=\left\{\phi_{k}\right\}$,

$$
\left|\prod_{k=1}^{n} a_{k}\right|^{1 / n} \leq \phi_{n}^{-1}\left(\frac{1}{n} \rho_{n}(\Phi, a)\right) \text { for all } n \text {. }
$$

PROOF. By the arithmetic-geometric mean inequality and Jensen's inequality,

$$
\phi_{n}\left(\left|\prod_{k=1}^{n} a_{k}\right|^{1 / n}\right) \leq \phi_{n}\left(\frac{1}{n} \sum_{k=1}^{n}\left|a_{k}\right|\right) \leq \frac{1}{n} \sum_{k=1}^{n} \phi_{k}\left(\left|a_{k}\right|\right)=\frac{1}{n} \rho_{n}(\Phi, a) .
$$

LEMMA 1.5. Given sequences $a=\left\{a_{k}\right\}$ and $b=\left\{b_{k}\right\}$, and $\Phi^{*}$-sequences $\Phi=$ $\left\{\phi_{n}\right\}$ and $\Psi=\left\{\psi_{n}\right\}$, for every $n$ there is a $k_{0}, 1 \leq k_{0} \leq n$, such that

$$
\left|a_{k_{0}} b_{k_{0}}\right| \leq \phi_{n}^{-1}\left(\frac{1}{n} \rho_{n}(\Phi, a)\right) \psi_{n}^{-1}\left(\frac{1}{n} \rho_{n}(\Psi, b)\right) .
$$

PROOF. Let $k_{0}$ be such that $\left|a_{k_{0}} b_{k_{0}}\right|=\min \left\{\left|a_{k} b_{k}\right|: 1 \leq k \leq n\right\}$. Then

$$
\begin{aligned}
\left|a_{k_{0}} b_{k_{0}}\right| & \leq\left|\prod_{k=1}^{n} a_{k} b_{k}\right|^{1 / n}=\left(\left|\prod_{k=1}^{n} a_{k}\right|\right)^{1 / n}\left(\left|\prod_{k=1}^{n} b_{k}\right|\right)^{1 / n} \\
& \leq \phi_{n}^{-1}\left(\frac{1}{n} \rho_{n}(\Phi, a)\right) \psi_{n}^{-1}\left(\frac{1}{n} \rho_{n}(\Psi, b)\right)
\end{aligned}
$$

by Lemma 1.4 .

We now state the first of our major preliminary results. 
THEOREM 1.6. Let $\Phi=\left\{\phi_{k}\right\}$ and $\Psi=\left\{\psi_{k}\right\}$ be $\Phi^{*}$-sequences and $a=\left\{a_{k}\right\}$ and $b=\left\{b_{k}\right\}$ sequences of real numbers. Then

$$
\begin{aligned}
\left|\sum_{k=1}^{n} \sum_{i=1}^{k} a_{i} b_{k}\right| \leq & \phi_{1}^{-1}\left(\rho_{1}^{*}(\Phi, a)\right) \psi_{1}^{-1}\left(\rho_{1}^{*}(\Psi, b)\right) \\
& +\sum_{k=1}^{n-1} \phi_{k}^{-1}\left(\frac{1}{k} \rho_{k}^{*}(\Phi, a)\right) \psi_{k}^{-1}\left(\frac{1}{k} \rho_{k}^{*}(\Psi, b)\right)
\end{aligned}
$$

(where the sum is taken to be 0 when $n=1$ ).

PROOF. We proceed by induction:

For $n=1$, we have

$$
\begin{aligned}
\left|a_{1} b_{1}\right| & =\phi_{1}^{-1}\left(\phi_{1}\left(\left|a_{1}\right|\right)\right) \psi_{1}^{-1}\left(\psi_{1}\left(\left|b_{1}\right|\right)\right) \\
& \leq \phi_{1}^{-1}\left(\rho_{1}^{*}(\Phi, a)\right) \psi_{1}^{-1}\left(\rho_{1}^{*}(\Psi, b)\right) .
\end{aligned}
$$

For $n>1$, define a sequence $a^{\prime}=\left\{a_{k}^{\prime}\right\}$ by $a_{k}^{\prime}=a_{k+1}$. Then by Lemma 1.5 , there is a $k_{0}$ with $1 \leq k_{0} \leq n-1$ so that

$$
\left|a_{k_{0}}^{\prime} b_{k_{0}}\right|=\left|a_{k_{0}+1} b_{k_{0}}\right| \leq \phi_{n-1}^{-1}\left(\frac{1}{n-1} \rho_{n-1}\left(\Phi, a^{\prime}\right)\right) \psi_{n-1}^{-1}\left(\frac{1}{n-1} \rho_{n-1}(\Psi, b)\right) \text {. }
$$

Now $\rho_{n-1}\left(\Phi, a^{\prime}\right) \leq \rho_{n-1}^{*}\left(\Phi, a^{\prime}\right) \leq \rho_{n-1}^{*}(\Phi, a)$ and $\rho_{n-1}(\Psi, b) \leq \rho_{n-1}^{*}(\Psi, b)$, so that

$$
\left|a_{k_{0}+1} b_{k_{0}}\right| \leq \phi_{n-1}^{-1}\left(\frac{1}{n-1} \rho_{n-1}^{*}(\Phi, a)\right) \psi_{n-1}^{-1}\left(\frac{1}{n-1} \rho_{n-1}^{*}(\Psi, b)\right) .
$$

Define sequences $c=\left\{c_{k}\right\}$ and $d=\left\{d_{k}\right\}$ as follows:

$$
c_{k}=\left\{\begin{array}{ll}
a_{k}, & k<k_{0}, \\
a_{k_{0}}+a_{k_{0}+1}, & k=k_{0}, \\
a_{k+1}, & k>k_{0},
\end{array} \quad \text { and } \quad d_{k}= \begin{cases}b_{k}, & k<k_{0}, \\
b_{k_{0}}+b_{k_{0}+1}, & k=k_{0}, \\
b_{k+1}, & k>k_{0},\end{cases}\right.
$$

and note that $\rho_{n}^{*}(\Phi, c) \leq \rho_{n}^{*}(\Phi, a)$ and $\rho_{n}^{*}(\Psi, d) \leq \rho_{n}^{*}(\Psi, b)$. A straightforward calculation shows that

$$
\sum_{k=1}^{n-1} \sum_{i=1}^{k} c_{i} d_{k}=a_{k_{0}+1} b_{k_{0}}+\sum_{k=1}^{n} \sum_{i=1}^{k} a_{i} b_{k}
$$

so we have

$$
\begin{aligned}
\left|\sum_{k=1}^{n} \sum_{i=1}^{k} a_{i} b_{k}\right| \leq & \left|\sum_{k=1}^{n-1} \sum_{i=1}^{k} c_{i} d_{k}\right|+\left|a_{k_{0}+1} b_{k_{0}}\right| \\
\leq & \phi_{1}^{-1}\left(\rho_{1}^{*}(\Phi, c)\right) \psi_{1}^{-1}\left(\rho_{1}^{*}(\Psi, d)\right) \\
& +\sum_{k=1}^{n-2} \phi_{k}^{-1}\left(\frac{1}{k} \rho_{k}^{*}(\Phi, c)\right) \psi_{k}^{-1}\left(\frac{1}{k} \rho_{k}^{*}(\Psi, d)\right) \\
& +\phi_{n-1}^{-1}\left(\frac{1}{n-1} \rho_{n-1}^{*}(\Phi, a)\right) \psi_{n-1}^{-1}\left(\frac{1}{n-1} \rho_{n-1}^{*}(\Psi, b)\right) \\
\leq & \phi_{1}^{-1}\left(\rho_{1}^{*}(\Phi, a)\right) \psi_{1}^{-1}\left(\rho_{1}^{*}(\Psi, b)\right) \\
& +\sum_{k=1}^{n-1} \phi_{k}^{-1}\left(\frac{1}{k} \rho_{k}^{*}(\Phi, a)\right) \psi_{k}^{-1}\left(\frac{1}{k} \rho_{k}^{*}(\Psi, b)\right)
\end{aligned}
$$


We now examine Riemann-Stieltjes sums. Let $P=\left\{x_{k}\right\}_{k=0}^{n}$ be a partition of $[a, b]$, and $\Xi=\left\{\xi_{k} \mid 1 \leq k \leq n ; x_{k-1} \leq \xi_{k} \leq x_{k}\right\}$ a collection of intermediate points. We write

$$
S(f, g ; P, \Xi)=\sum_{k=1}^{n} f\left(\xi_{k}\right)\left(g\left(x_{k}\right)-g\left(x_{k-1}\right)\right) .
$$

THEOREM 1.7. Let $\Phi=\left\{\phi_{k}\right\}$ and $\Psi=\left\{\psi_{k}\right\}$ be $\Phi^{*}$-sequences, and $P$ and $\Xi$ be as above with $n$ the number of intervals in $P$. If $f(a)=0$, the following estimate holds:

$$
|S(f, g ; P, \Xi)| \leq 2 \sum_{k=1}^{n-1} \phi_{k}^{-1}\left(\frac{1}{k} V_{\Phi}(f)\right) \psi_{k}^{-1}\left(\frac{1}{k} V_{\Psi}(g)\right) .
$$

ProOF. Take $\xi_{0}=a$; then

$$
\begin{aligned}
|S(f, g ; P, \Xi)| & =\left|\sum_{k=1}^{n} f\left(\xi_{k}\right)\left(g\left(x_{k}\right)-g\left(x_{k-1}\right)\right)\right| \\
& =\left|\sum_{k=1}^{n} \sum_{i=1}^{k}\left(f\left(\xi_{i}\right)-f\left(\xi_{i-1}\right)\right)\left(g\left(x_{k}\right)-g\left(x_{k-1}\right)\right)\right| .
\end{aligned}
$$

Now take $\alpha_{i}=f\left(\xi_{i}\right)-f\left(\xi_{i-1}\right), i=1, \ldots, n, \alpha_{i}=0$ for $i>n$ and $\beta_{k}=g\left(x_{k}\right)-$ $g\left(x_{k-1}\right), k=1, \ldots, n, \beta_{k}=0$ for $k>n$, and let $\alpha=\left\{\alpha_{i}\right\}$ and $\beta=\left\{\beta_{i}\right\}$. Then the above

$$
\begin{aligned}
& =\left|\sum_{k=1}^{n} \sum_{i=1}^{k} \alpha_{i} \beta_{k}\right| \\
& \leq \phi_{1}^{-1}\left(\rho_{1}^{*}(\Phi, \alpha)\right) \psi_{1}^{-1}\left(\rho_{1}^{*}(\Psi, \beta)\right)+\sum_{k=1}^{n-1} \phi_{k}^{-1}\left(\frac{1}{k} \rho_{k}^{*}(\Phi, \alpha)\right) \psi_{k}^{-1}\left(\frac{1}{k} \rho_{k}^{*}(\Psi, \beta)\right) \\
& \leq \phi_{1}^{-1}\left(V_{\Phi}(f)\right) \psi_{1}^{-1}\left(V_{\Psi}(g)\right)+\sum_{k=1}^{n-1} \phi_{k}^{-1}\left(\frac{1}{k} V_{\Phi}(f)\right) \psi_{k}^{-1}\left(\frac{1}{k} V_{\Psi}(g)\right) \\
& \leq 2 \sum_{k=1}^{n-1} \phi_{k}^{-1}\left(\frac{1}{k} V_{\Phi}(f)\right) \psi_{k}^{-1}\left(\frac{1}{k} V_{\Psi}(g)\right) \cdot
\end{aligned}
$$

We now prove the main result of this chapter.

THEOREM 1.8. Let $\Phi=\left\{\phi_{n}\right\}$ and $\Psi=\left\{\psi_{n}\right\}$ be $\Phi$-sequences such that

$$
\sum_{n} \phi^{-1}(1 / n) \psi_{n}^{-1}(1 / n)<\infty
$$

If $f \in \Phi \mathrm{BV}_{0} \cap C$ and $g \in \Psi \mathrm{BV}$, then $\int_{a}^{b} f d g$ exists.

Proof. We may assume that $V_{\Phi}(f)<\infty$ and $V_{\Psi}(g)<\infty$. Let $P_{1}=\left\{x_{k}^{1}\right\}_{k=0}^{n_{1}}$ and $P_{2}=\left\{x_{k}^{2}\right\}_{k=0}^{n_{2}}$ be partitions of $[a, b]$ with sets of intermediate points $\Xi_{1}=\left\{\xi_{k}^{1}\right\}$ and $\Xi_{2}=\left\{\xi_{k}^{2}\right\}$ respectively, and let $P=\left\{x_{k}\right\}_{k=0}^{n}$ be the "common refinement" of $P_{1}$ and $P_{2}$. Define step functions $f_{1}$ and $f_{2}$ by

$$
f_{i}(x)=f\left(x ; P_{i}, \Xi_{i}\right)=\left\{\begin{array}{ll}
0, & x=a, \\
f\left(\xi_{k}^{i}\right), & x_{k-1}^{i}<x \leq x_{k}^{i},
\end{array} \quad \text { for } i=1,2 .\right.
$$


Then, for $i=1,2$, we have

$$
\begin{aligned}
S\left(f, g ; P_{i}, \Xi_{i}\right) & =\sum_{k=1}^{n_{i}} f\left(\xi_{k}^{i}\right)\left(g\left(x_{k}^{i}\right)-g\left(x_{k-1}^{i}\right)\right) \\
& =\sum_{k=1}^{n_{i}} f_{i}\left(x_{k}^{i}\right)\left(g\left(x_{k}^{i}\right)-g\left(x_{k-1}^{i}\right)\right) \\
& =\sum_{k=1}^{n} f_{i}\left(x_{k}\right)\left(g\left(x_{k}\right)-g\left(x_{k-1}\right)\right)
\end{aligned}
$$

so that

$$
S\left(f, g ; P_{1}, \Xi_{1}\right)-S\left(f, g ; P_{2}, \Xi_{2}\right)=2 \sum_{k=1}^{n} \frac{1}{2}\left(f_{1}\left(x_{k}\right)-f_{2}\left(x_{k}\right)\right)\left(g\left(x_{k}\right)-g\left(x_{k-1}\right)\right),
$$

the factors of 2 and $\frac{1}{2}$ being for later convenience. The above sum

$$
=2 S\left(\frac{1}{2}\left(f_{1}-f_{2}\right), g ; P, P\right) \text {. }
$$

Applying Theorem 1.7 to the functions $\frac{1}{2}\left(f_{1}-f_{2}\right)$ and $g$ and the $\Phi^{*}$-sequences $F \Phi=\left\{F \Phi_{n}\right\}$ and $F \Psi=\left\{F \Psi_{n}\right\}$, the function $F$ having been chosen in Lemma 1.2, we have

$$
\begin{aligned}
& \left|S\left(f, g ; P_{1}, \Xi_{1}\right)-S\left(f, g ; P_{2}, \Xi_{2}\right)\right| \\
& \quad \leq 4 \sum_{k=1}^{n}\left(F \phi_{k}\right)^{-1}\left(\frac{1}{k} V_{F \Phi}\left(\frac{1}{2}\left(f_{1}-f_{2}\right)\right)\right)\left(F \psi_{k}\right)^{-1}\left(\frac{1}{k} V_{F \Psi}(g)\right) .
\end{aligned}
$$

By Lemma 1.3,

$$
\begin{aligned}
V_{F \Phi}\left(\frac{1}{2}\left(f_{1}-f_{2}\right)\right) & \leq \chi_{1}\left(\Phi, B\left(\frac{1}{2}\left(f_{1}-f_{2}\right)\right)\right) V_{\Phi}\left(\frac{1}{2}\left(f_{1}-f_{2}\right)\right) \\
& \leq \frac{1}{2} \chi_{1}\left(\Phi, B\left(\frac{1}{2}\left(f_{1}-f_{2}\right)\right)\right)\left(V_{\Phi}\left(f_{1}\right)+V_{\Phi}\left(f_{2}\right)\right) \\
& \leq \chi_{1}\left(\Phi, B\left(\frac{1}{2}\left(f_{1}-f_{2}\right)\right)\right) V_{\Phi}(f) \\
& \leq \chi_{1}\left(\Phi, B\left(\frac{1}{2}\left(f_{1}-f\right)\right)+B\left(\frac{1}{2}\left(f_{2}-f\right)\right)\right) V_{\Phi}(f),
\end{aligned}
$$

and note again that $V_{F \Psi}(g)<\infty$, since $g \in \Psi \mathrm{BV}$. Since $f$ is uniformly continuous, for $\varepsilon>0$ given, we may choose $\delta>0$ so that $B\left(\frac{1}{2}\left(f_{1}-f\right)\right)+B\left(\frac{1}{2}\left(f_{2}-f\right)\right)<\varepsilon$ for $\mu\left(P_{1}\right), \mu\left(P_{2}\right)<\delta$. Then

$$
\begin{aligned}
& \left|S\left(f, g ; P_{1}, \Xi_{1}\right)-S\left(f, g ; P_{2}, \Xi_{2}\right)\right| \\
& \quad \leq \sum_{k=1}^{\infty}\left(F \phi_{k}\right)^{-1}\left(\frac{1}{k} \chi_{1}(\Phi, \varepsilon) V_{\Phi}(f)\right)\left(F \psi_{k}\right)^{-1}\left(\frac{1}{k} V_{F \Psi}(g)\right),
\end{aligned}
$$

which converges for $\varepsilon>0$ by Lemmas 1.1 and 1.2 . Since $\chi_{1}(\Phi, \varepsilon) \rightarrow 0$ as $\varepsilon \rightarrow 0$, we may make this sum as small as we wish by choosing $\varepsilon$ sufficiently small.

COROllary 1.9. Let $\Lambda=\left\{\lambda_{k}\right\}$ and $\Gamma=\left\{\gamma_{k}\right\}$ be $\Lambda$-sequences such that $\sum_{k} \lambda_{k} \gamma_{k} / k^{2}<\infty$. For $f \in \Lambda \mathrm{BV}_{0} \cap C$ and $g \in \Gamma \mathrm{BV}_{0}$, the following estimate holds:

$$
\left|\int_{a}^{b} f d g\right| \leq 2\|f\|_{\Lambda}\|g\|_{\Gamma} \sum_{k} \lambda_{k} \gamma_{k} / k^{2}
$$

where $\|f\|_{\Lambda}=|f(a)|+V_{\Lambda}(f)$. 


\section{BIBLIOGRAPHY}

1. R. Leśniewicz and W. Orlicz, On generalized variations (II), Studia Math. 45 (1973), 71-109.

2. M. Schramm and D. Waterman, On the magnitude of Fourier coefficients, Proc. Amer. Math. Soc. 85 (1982), 407-410.

3. __ Absolute convergence of Fourier series of functions of $\Lambda \mathrm{BV}^{(P)}$ and $\phi \Lambda \mathrm{BV}$, Acta Math. Hungary 40 (1982), 273-276.

4. D. Waterman, On convergence of Fourier series of functions of generalized bounded variation, Studia Math. 44 (1972), 107-117.

5. __, On $\Lambda$-bounded variation, Studia Math. 57 (1976), 33-45.

6. L. C. Young, An inequality of Hölder type connected with Stieltjes integration, Acta Math. 67 (1936), 251-282.

7. __ Sur une généralisation de la notion de variation de puissance p-ième bornée au sens de $M$. Wiener, et sur la convergence des séries de Fourier, Comptes Rendus 204 (1937), 470-472.

Department of mathematics, hamilton College, Clinton, New York 13323 